\title{
Nonequilibrium pattern formation in chiral Langmuir monolayers with transmembrane flows
}

\author{
Tatsuo Shibata ${ }^{1}$ and Alexander S. Mikhailov ${ }^{2}$ \\ ${ }^{1}$ Department of Mathematics and Life Sciences, Hiroshima University, \\ 1-3-1, Kagamiyama, Higashi-Hiroshima, 739-8526, Japan, \\ and ${ }^{2}$ Abteilung Physikalische Chemie, Fritz-Haber-Institut der \\ Max-Planck-Gesellschaft, Faradayweg 4-6, 14195 Berlin, Germany
}

\begin{abstract}
Nonequilibrium Langmuir monolayers including a fraction of chiral molecules and subject to transmembrane flow are considered. The flow induces coherent collective precession of chiral molecules. Our theoretical study shows that splay interactions in this system lead to spatial redistribution of chiral molecules and formation of spiral waves and target patterns observed in experiments.
\end{abstract}

PACS numbers: $82.40 . \mathrm{Np}, 68.18 . \mathrm{g}, 82.40 . \mathrm{Np}$

Studies of pattern formation in nonequilibrium soft matter are essential for understanding the operation of biological cells and for potential applications [1]. Biological membranes including active molecular pumps or channels can develop shape oscillations and show persistent wave propagation [2, 3]. If a membrane contains rotating molecular motors, interactions between them may lead to the development of regular arrays with the crystalline order 4]. Phase separation in two-component lipid layers is responsible for budding and replication of vesicles [5, 6]. Closely related to biomembranes, Langmuir monolayers are formed by organic lipid or amphiphilic molecules disposed on a liquid-gas interface [7]. Nonequilibrium patterns of traveling orientation waves in illuminated two-component Langmuir monolayers, where illumination leads to transitions between different conformational states of molecules, have been experimentally and theoretically investigated [8, 9, 10, 11]. Recently, Tabe and Yokoyama have demonstrated that Langmuir liquid-crystal monolayers including chiral molecules ("molecular rotors") are easily brought to and maintained at nonequilibrium conditions by transmembrane flows 12. If there is a gradient of small molecules across a Langmuir monolayer, i.e. their concentrations in the liquid and the gas are different, this produces a flow of such molecules through the monomolecular layer. Such transmembrane flow gives rise to coherent collective precession of molecular rotors. Experiments using reflectedlight polarizing microscopy have revealed that the precession is not uniform and complex orientational wave patterns are observed. This behavior is apparently universal; it has been verified for a number of chiral chemicals and different experimental conditions 12].

In this Letter, we construct a phenomenological theory of spatiotemporal pattern formation in chiral Langmuir monolayers with transmembrane flows. An important role in such systems is played by splay coupling between local concentration of chiral molecules and the orientational field 13, 14, 15]. We show that, in the presence of transmembrane flow, this coupling gives rise to nonequilibrium wave patterns in the orientational field and spatial redistribution of chiral molecules inside the monolayer. The target patterns, seen in the experiments [12], should thus be accompanied by aggregation of chiral molecules in the periphery of the patterns. Other wave patterns, such as traveling stripes and rotating spiral waves, are also possible.

We study a model of an orientationally ordered twocomponent Langmuir monolayer representing a mixture of chiral and achiral molecules (in the experiments 12 . the chiral molecules were making up only $10 \%$ of the monolayer). The local state of the monolayer is described by the variable $c$, giving the local fraction of chiral molecules, and by the director vector $\mathbf{n}$ that represents the projection of the molecular tilt onto the monolayer plane. The Landau free energy of the system is

$$
\begin{gathered}
F=\int\left[\frac{1}{2} K(\nabla \mathbf{n})^{2}+k_{B} T c \ln c+k_{B} T(1-c) \ln (1-c)\right. \\
\left.+\frac{1}{2} G(\nabla c)^{2}+\Lambda c \nabla \cdot \mathbf{n}\right] d x d y .
\end{gathered}
$$

The first term corresponds to the elastic energy of orientational ordering ( $K$ is the Frank elastic constant). The next two terms determine the lattice-gas entropy contribution to the free energy ( $T$ is the temperature and $k_{B}$ is the Boltzmann constant), and the following term (with the coefficient $G$ ) takes into account weak energetic interactions between chiral molecules which favor their uniform spatial distribution. The last term in the expression for free energy describes splay interactions in the system. It provides coupling between the scalar concentration field $c$ and the vector orientational field $\mathbf{n}$ [13]; the parameter $\Lambda$ specifies the strength of splay interactions [16].

The kinetic equation for the local concentration $c$ of chiral molecules is

$$
\dot{c}=\frac{D}{k_{B} T} \nabla\left[c(1-c) \nabla \frac{\delta F}{\delta c(\mathbf{r}, t)}\right]
$$

where $D$ is their diffusion constant. The kinetic equations for the director field $\mathbf{n}$ are

$$
\dot{n}_{x}=-\Gamma \frac{\delta F}{\delta n_{x}(\mathbf{r}, t)}+\Omega n_{y}, \quad \dot{n}_{y}=-\Gamma \frac{\delta F}{\delta n_{y}(\mathbf{r}, t)}-\Omega n_{x} .
$$


In addition to the relaxation terms ( $\Gamma$ is the relaxation rate constant for orientational ordering), we have phenomenologically included into these equations, following Ref. 12], a term that describes planar precession of the director vector. This precession is caused by the transmembrane flow and its frequency $\Omega$ is linearly proportional to the flow intensity (as seen in the experiments 12]). Because of the flow terms, the system cannot relax to the state of thermal equilibrium and oscillations and active wave propagation become possible.

Rescaling time and spatial coordinates as $t \rightarrow$ $t\left(k_{B} T \Gamma\right)^{-1}$ and $\mathbf{r} \rightarrow \mathbf{r}\left(K / k_{B} T\right)^{1 / 2}$ and using the angle variable $\phi$ defined by $\mathbf{n}=(\cos \phi, \sin \phi)$, kinetic equations (21) and (3) can be written in the form

$$
\dot{c}=\nu\left[\nabla^{2} c-g \nabla\left(c(1-c) \nabla^{3} c\right)+\lambda \nabla(c(1-c) \nabla(\nabla \cdot \mathbf{n})],\right.
$$

$$
\dot{\phi}=\nabla^{2} \phi-\omega+\lambda\left(\cos \phi \frac{\partial c}{\partial y}-\sin \phi \frac{\partial c}{\partial x}\right) .
$$

The coefficients in these equations are $\nu=D(K \Gamma)^{-1}, g=$ $G K^{-1}, \lambda=\Lambda /\left(k_{B} T K\right)^{1 / 2}$ and $\omega=\Omega\left(k_{B} T \Gamma\right)^{-1}$. Note that the total amount of chiral molecules is conserved and average spatial concentration $c_{0}$ of these molecules is a parameter of the system. According to equation (4), splay coupling to the director field leads to physical forces acting on chiral molecules and to the viscous flow of these molecules in the monolayer plane. On the other hand, spatial gradients of concentration $c$ lead, according to equation (5), to local rotation of the director vector $\mathbf{n}$.

When transmembrane flow is absent $(\omega=0)$, these kinetic equations describe relaxation to thermal equilibrium. The stationary equilibrium state is uniform if splay interactions are sufficiently weak. If the splay interaction strength $\lambda$ exceeds the critical value $\lambda_{c r}=$ $\left[c_{0}\left(1-c_{0}\right)\right]^{-1 / 2}$, the uniform state becomes however unstable with respect to growth of spatial modes with the wavenumbers $0<k<k_{\max }$, where $k_{\max }^{2} \propto\left(\lambda-\lambda_{c r}\right) / g$. This instability has previously been investigated and is known to lead to the formation of an equilibrium periodic stripe pattern [13]. In this equilibrium pattern, both the local concentration and the director orientation are periodically varying along a certain direction.

To investigate nonequilibrium pattern formation induced by transmembrane flow, numerical simulations of the model (4) and (5) using the explicit Euler scheme with constant coordinate and time steps were performed. In all simulations, periodic boundary conditions were applied.

When the transmembrane flow is introduced $(\omega \neq 0)$, we see that the equilibrium stationary stripe pattern begins to move at a velocity that increases with the flux intensity $\omega$. Figure 1 shows profiles of concentration and azimuthal angle across a traveling stripe pattern. Note that the shape of the stripes and their spatial period are

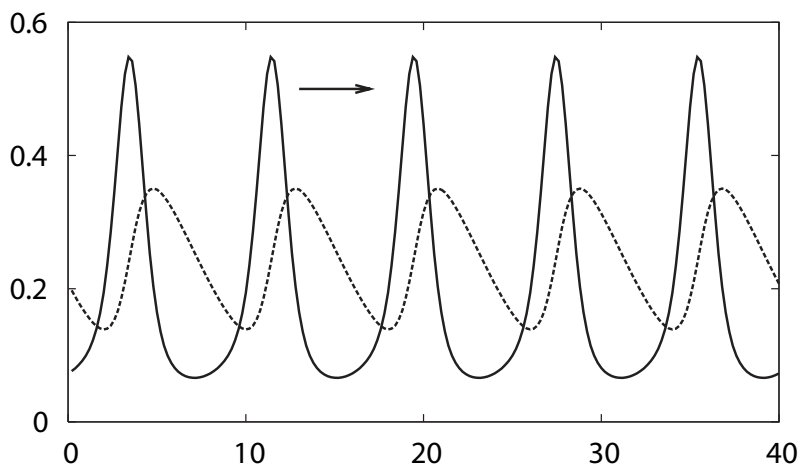

FIG. 1: Profiles of concentration $c$ (solid) and azimuthal angle $\phi / 2 \pi$ (dashed) in a traveling stripe pattern for $c_{0}=$ $0.2, \nu=0.1, g=1, \lambda=3$, and $\omega=0.005$. The arrow shows the direction of motion.

not significantly different from the respective equilibrium pattern at $\omega=0$.

If a simulation is started with random initial conditions for the azimuthal angle field $\phi$, regular stripes are not formed. Instead, the system undergoes relaxation to an equilibrium state with many spiral-shaped orientational defects. Application of the transmembrane flow to a system in this state leads, after a transient, to complex self-organized wave patterns. Several examples of such patterns for different parameter values are shown in Fig 2 and movies [18].

The central region in the pattern shown in Fig.2a is periodically emitting orientation waves. Repeated generation and outward propagation of these waves is seen in the space-time diagram Fig 3 which displays evolution of the azimuthal angle distribution along the diagonal cross section indicated by the dashed line in Fig.2a. The waves spread out in the large central region and run into the periphery part of the pattern, occupied by stripes with a shorter wavelength. The corners of the medium in Fig.2a are occupied by spiral-shaped stripe structures (because periodic boundary conditions are used, they represent four parts of the same compact pattern). This stripe structure represents a wave sink, as can be seen from the space-time diagram in Fig 3 (the sink occupies the upper part of this diagram) and by examining the respective movie [18]. While a target pattern is seen in the center for the azimuthal angle distributions (right panel), a spiral wave occupying the central region is seen in the concentration distribution (left panel in Fig. 2a).

Figure 4a displays a superposition of three subsequent snapshots of concentration profiles of chiral molecules along the diagonal line in Fig. 2a. Additionally, we show here the concentration profile $\bar{c}(x)$ smoothed over the spatial scale $\Delta x=56$ (roughly the stripe period) and averaged over the time interval of 10000. Although spatial variations are substantial, we find that, on the average, the central region of the target pattern is de- 

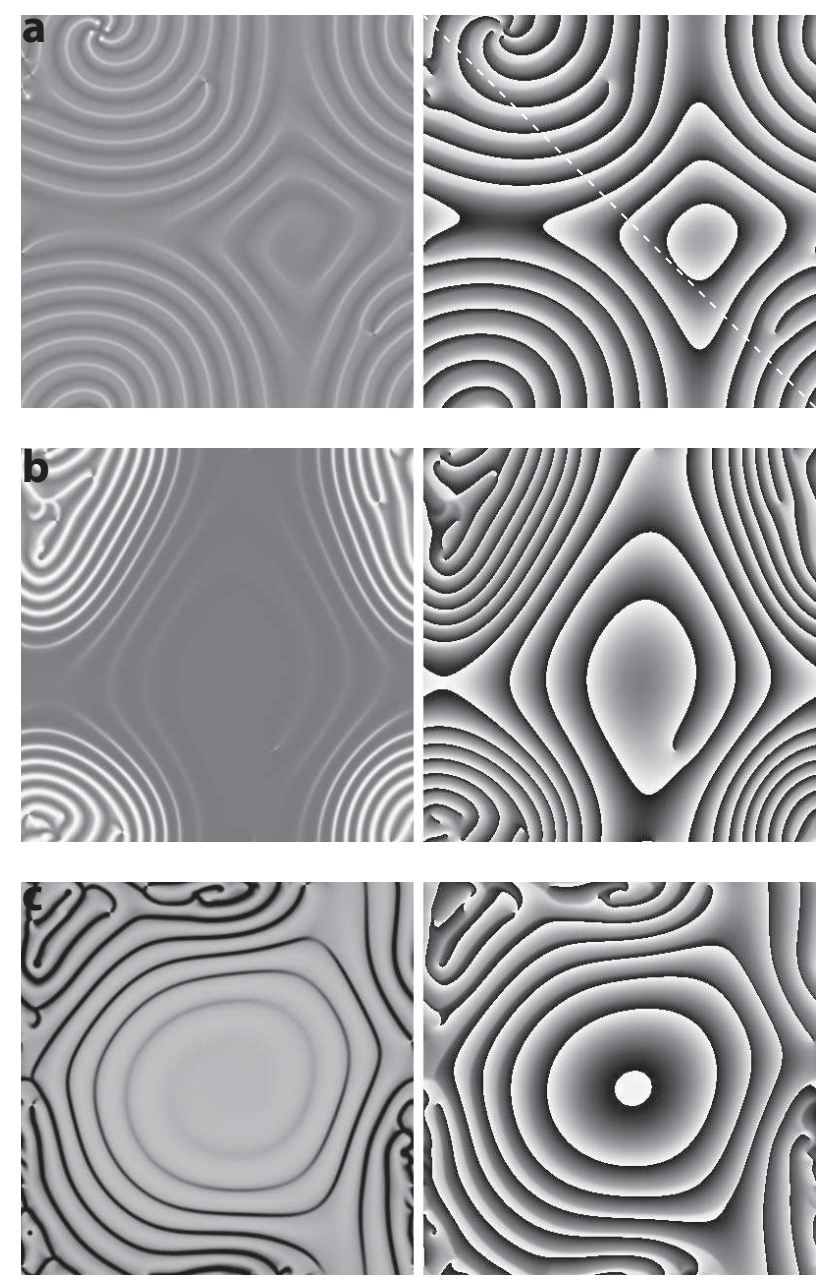

FIG. 2: Distributions of concentration (left panels) and azimuthal angle (right panels) in self-organized wave patterns obtained starting from random initial conditions for systems with the parameters: (a) $c_{0}=0.1, \lambda=3.4, \omega=0.01, \nu=$ $10, g=10$ and (b) $c_{0}=0.1, \lambda=3, \omega=0.015 \nu=0.01, g=1$ and (c) $c_{0}=0.9, \lambda=3.4, \omega=0.015 ., \nu=0.01, g=10$, The linear size of the medium is $L=800$ (a) and $L=200$ (b and c). The concentration is displayed in gray scale with the darker color corresponding to lower concentration values.

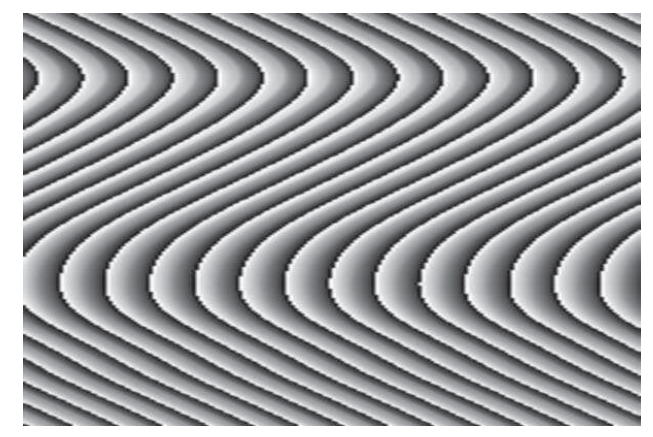

FIG. 3: Space-time diagrams displaying evolution of the azimuthal angle along the diagonal cross section shown by dashed line in Fig.2a. Time runs from left to right in the horizontal direction, the total shown time interval is $T=10000$. The target pattern and the wave sink (above) are seen.
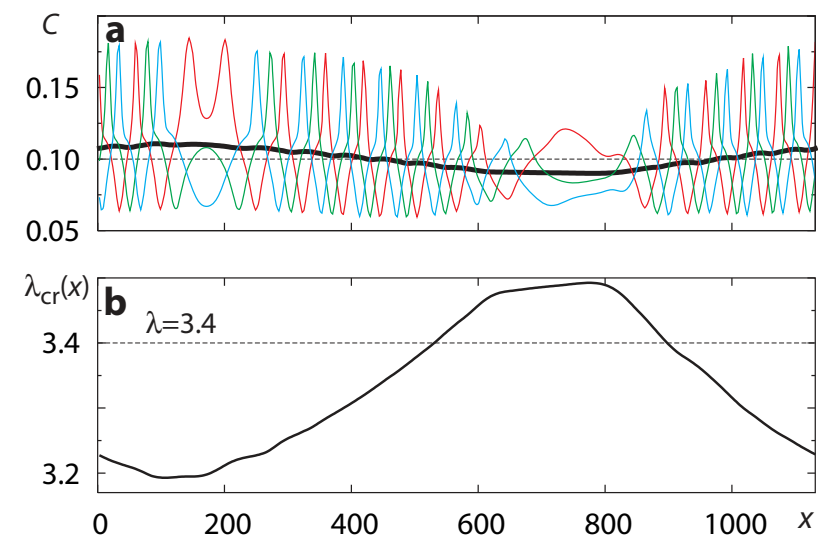

FIG. 4: (a) Snapshots of concentration profiles $c(x, t)$ along the diagonal dashed line in Fig.2a for three subsequent time moments (color online: red, green, blue); the black solid curve shows the average smooth profile $\bar{c}(x)$. (b) Effective local critical splay interaction $\lambda_{c r}(x)$ along the same line.

pleted of chiral molecules which become concentrated in the curled stripes in its periphery.

The spatial redistribution of chiral molecules allows to qualitatively explain the emergence of target-shaped wave patterns. When a stripe pattern, caused by splay interactions, is formed, it tends to hinder the angular rotation forced by the transmembrane flow. Therefore, oscillations develop only in the areas free from the stripes. As we have seen above, the critical splay interaction strength, needed for the formation of stripes, depends on the concentration as $\lambda_{c r}(c)=[c(1-c)]^{-1 / 2}$. If the concentration is not constant and smoothly varies in space, the critical conditions will be determined by the local concentration. As seen from Fig.4a, the concentration varies rather rapidly inside the stripe regions. If we smooth the concentration profile and substitute $\bar{c}(x)$ instead of $c$ in the expression for the critical splay interaction strength, we obtain the effective dependence $\lambda_{c r}(x)$ displayed in Fig. 4b. For comparison, the horizontal dash line shows the value of $\lambda$ in the respective simulation. Thus, inside the central area we have $\lambda<\lambda_{c r}(x)$, which explains why stripes are absent here. The boundary where the stripes first develop is roughly determined by the condition $\lambda<\lambda_{c r}(x)$. In the region filled with stripes, $\lambda_{c r}(x)$ lies below $\lambda$, so that the uniform state is unstable. Of course, this argument is only approximate because the stripes are not at equilibrium. However, the effect of transmembrane flow on the stripe pattern is relatively weak, as we have already noticed.

The simulation shown in Fig. 2a has been performed assuming strong diffusion of chiral molecules $(\nu=10)$. When diffusion is weaker $(\nu=0.01$ in Fig. 2b), depletion of chiral molecules in the central region and their accumulation inside the stripe structure in the periphery become strongly pronounced. Now, the characteristic 
wavelength of the stripes in the periphery of the target pattern is much shorter than the wavelength of the equilibrium stripe pattern for $c=c_{0}$. This is because the wavelength of the stripe pattern decreases when the difference $\left|\lambda-\lambda_{c r}(x)\right|$ becomes larger. The stripes move only very slowly in the periphery under these conditions. The large central region can contain rotating spiral waves, as seen in Fig. 2b.The splay intensity strength $\lambda=3$ in this simulation was below the critical strength $\lambda_{c r}=3.33 \cdots$ corresponding to $c_{0}=0.1$. This means that the uniform state remains stable with respect to small perturbations. However, strong initial perturbations can still lead in this case to the formation of steady wave patterns. The spiralshaped stripe pattern in Fig. 2b is similar to the equilibrium spiral patterns 17]. Note that a number of topological orientational defects are present in the pattern shown in Fig. 2a, but all of them, except one, belong to the dense stripe region in the periphery.

So far, only patterns for low average concentrations of chiral molecules have been discussed. In contrast to this, the wave pattern shown in Fig. 2c corresponds to a high concentration of chiral molecules $\left(c_{0}=0.9\right)$. Analyzing this pattern, several significant differences are seen. The concentration of chiral molecules is now increased inside the uniform central region and decreased in the region occupied by the stripes. The difference in the spatial distribution of chiral molecules for $c_{0}=0.9$ can be explained if we notice that $\lambda_{c r}(c)=[c(1-c)]^{-1 / 2}$ depends non-monotonously on concentration $c$ and increases with concentration when $c>0.5$. Therefore, inside the central region the formation of stripes is prevented because the condition $\lambda<\lambda_{c r}(x)$ again holds, now because the chiral molecules have aggregated in this region, pushing the achiral component into the stripe-filled periphery region.

Thus, we have shown that splay interactions, based on coupling between the orientational and concentration fields, determine principal properties of nonequilibrium wave patterns in chiral Langmuir monolayers subject to the transmembrane flow. The theory explains targetshaped and spiral wave patterns observed in the experiments [12, 19]. It relates the formation of such patterns to nonequilibrium spatial redistribution of molecular rotors, tending to aggregate in the areas occupied by slowly traveling, densely packed stripes.

Biomembranes are closely related to Langmuir monolayers and we expect that similar results should hold, under appropriate conditions, also for the membranes including a fraction of chiral molecules. The transmembrane flow in such systems is created by a gradient of concentration of small molecules or ions that leak through the membrane. The leakage may bring the membrane to nonequilibrium conditions, giving rise to traveling waves and complex self-organized wave patterns. Importantly, chiral molecules (and, possibly, some passive inclusions) can then be transported and spatially redistributed in a membrane as a result of wave propagation.

The authors acknowledge stimulating discussions with Y. Tabe and H. Yokoyama. Computer simulations were performed using a parallel supercomputer in the Yukawa Institute for Theoretical Physics. One of us (T.S.) acknowledges financial support through a grant for young scientists from the Ministry of Education, Culture, Sports, Science and Technology in Japan.

[1] A. S. Mikhailov and E. Ertl, Science, 267, 476 (1995)

[2] S. Ramaswamy, J. Toner, J. Prost, Phys. Rev. Lett. 84, 3494 (2000);

[3] H.-Y. Chen, Phys. Rev. Lett. 92, 168101 (2004)

[4] P. Lenz, J.-F. Joanny, F. Jülicher, J. Prost, Phys. Rev. Lett. 91, 108104 (2003)

[5] P. B. S. Kumar, G. Gompper, and R. Lipowsky, Phys. Rev. Lett. 86, 3911 (2001).

[6] K. Takakura and T. Sugawara, Langmuir 20, 3832 (2004)

[7] V. M. Kaganer, H. Möhwald, and P. Dutta, Rev. Mod. Phys.71, 779 (1999)

[8] Y. Tabe and H. Yokoyama, Langmuir 11, 4609 (1995).

[9] R. Reigada, F. Sagues, and A. S. Mikhailov, Phys. Rev. Lett. 89, 038301 (2002)

[10] R. Reigada, A. S. Mikhailov, and F. Sagues, Phys. Rev. E 69, 041103 (2004)

[11] T. Okuzono, Y. Tabe, and H. Yokoyama, Phys. Rev. E, 69, 050701 (2004)

[12] Y. Tabe and H. Yokoyama, Nature Materials, 2, 806 (2003)

[13] J. V. Selinger, Z. G. Wang, R. F. Bruinsma, and C. M. Knobler, Phys. Rev. Lett. 70, 1139 (1993)

[14] T. Ohyama, A. E. Jacobs, and D. Mukamel, Phys. Rev. E 532595 (1996)

[15] R. D. Kamien and J. V. Selinger, J. Phys.: Condens. Matter 13 R1 (2001)

[16] If a monolayer consists of chiral molecules, the splay term generally is $\Lambda c \nabla \cdot \mathbf{n}^{\prime}$ where $\mathbf{n}^{\prime}$ is obtained from $\mathbf{n}$ by rotation by some fixed angle $\alpha$. Since all other terms in the free energy are, however, invariant with respect to such rotation, it is not important for pattern formation and we put here $\alpha=0$.

[17] J. V. Selinger and R. L. Selinger, Phys. Rev. E 51, R860 (1995)

[18] The videos of simulations corresponding to Figures $2 \mathrm{a}$ and $2 \mathrm{~b}$ are reached at the homepage (http://home.hiroshima-u.ac.jp/shibata/Noneq/index.html).

[19] Rotating spiral waves have also been observed in the experiments (Y. Tabe, private communication). 